\section{Enseñanza del diseño asistido por computador en laFacultad de Ingeniería Industrial, UNMSM}

\author{
Recepción: Mayo de $2006 /$ Aceptación: Junio de 2006
}

(1) Oswaldo Rojas Lazo

(2) Julio Salas Bacalla

(3) Eulogio Santos De la Cruz

(4) Pedro Marín Chávez

(5) Ciro Mejía Elías

\section{RESUMEN}

La enseñanza del diseño asistido por computador en la Facultad de Ingeniería Industrial de la UNMSM, es el tema de este trabajo. Teniendo como referencia el plan de estudios, se analizan los cursos que pueden desarrollar dichos contenidos con o sin aplicación de software, se analizan los cuatro componentes básicos de un sistema CAD: software, hardware, humanware y base de datos.

\section{Palabras Clave: Enseñanza del CAD.}

\section{COMPUTER ASSISTED DESIGN (CAD)} EDUCATION AT THE INDUSTRIAL ENGINEERING FACULTY, UNMSM ABSTRACT

CAD teaching at the Faculty of Industrial Engineering of the UNMSM, is the subject of this work Having as a reference the curriculum, the courses are analyzed that can develop these contents with or without software application, the four basic components of a CAD system are analyzed: software, hardware, humanware and data base.

Key words: CAD Education.

\section{INTRODUCCIÓN}

El avance de la ciencia y tecnología en estos últimos años está transformando muchos factores de competitividad, como son los costos, calidad, tiempo, equipos, flexibilidad de los procesos, valor agregado, disponibilidad de equipos y tecnología, mano de obra, etc. En todos ellos una de las tendencias es más con la mente y menos con las manos. En el campo del diseño asistido por computador, el desarrollo se viene dando en función al hardware y software disponible, los precios del hardware y la disponibilidad del software permite que estos estén al alcance de las empresas, cuya aplicación dependerá del sector industrial, dimensión de la empresa, tecnología usada, etc, por lo que es imprescindible que nuestros alumnos salgan capacitados en el manejo de las tecnologías CAD.

En el año 2001 la Facultad de Ingeniería Industrial de la UNMSM se encontraba a la vanguardia en la enseñanza de estas tecnologías, actualmente se ha mantenido dicha vanguardia debido fundamentalmente a la falta de dirección de las autoridades y la poca dedicación de los profesores del área.

La curricula actual no está actualizada, mostrando carencias en el área relacionada a las tecnologías CAD/CAM, se espera que con la implementación del Centro de Manufactura Avanzada se pueda revertir dicha situación.

En el presente año se verá la actualización de curricula, buena oportunidad para realizar las modificaciones pertinentes.

\section{ANTECEDENTES}

En estos últimos años se han desarrollado vertiginosamente el hardware y software, lo que permite tener al alcance aplicaciones CAD que se pueden desarrollar en los distintas asignaturas.

Los trabajos realizados en la Facultad de Ingeniería Industrial relacionados con la tecnología CAD/CAM son:

- Tecnología moderna en un Laboratorio de Dibujo y Diseño (1991).

- Aplicación de software gráfico para ingenierías (1992).

(1) Ingeniero Industrial. Profesor del Departamento de Diseño y Tecnología Industrial, UNMSM. E-mail: orojasl@unmsm.edu.pe

(2) Ingeniero Industrial. Profesor del Departamento de Producción y Gestión Industrial, UNMSM. E-mail: jsalasb@unmsm.edu.pe

(3) Ingeniero Industrial. Profesor del Departamento de Diseño y Tecnología Industrial, UNMSM. E-mail: esantosd@unmsm.edu.pe

(4) Ingeniero Industrial. Profesor del Departamento de Diseño y Tecnología Industrial, UNMSM. E-mail:pmarinc@unmsm.edu.pe

(5) Ingeniero Industrial. Jefe de la Oficina de Estadística e Informática de la FII, UNMSM. E-mail: ofinfind@unmsm.edu.pe 
- Adaptación y enseñanza del Autocad al Dibujo Técnico (1993).

- Diseño e implementación de un laboratorio de producción automatizada CAD/CAM (1998).

En el año 1996 se crea el Círculo de Desarrollo para la tecnología CAD (CIDECAD), con el objetivo de reunir a alumnos y docentes que quieran desarrollar estas tecnologías.

En el año 2001 se inauguró el laboratorio CAD de la Facultad de Ingeniería Industrial, único en la UNMSM (15 computadoras PIII, 1 plotter, 2 software con sus respectivas licencias).

Los docentes del Departamento de Diseño y Tecnología Industrial (DADYTI) prestan servicios académicos a todas las demás facultades de ingenierías en los cursos de Dibujo y Diseño.

En los últimos 5 años los docentes del DADYTI no se han actualizado en lo referente a la tecnología CAD. Con el objetivo de incentivar el uso de estas nuevas tecnologías, se ha incluido a los profesores del Departamento de Diseño y Tecnología Industrial en este proyecto.

\section{ANTECEDENTES}

El estudio desarrollado es descriptivo y es una investigación exploratoria, se trata de especificar aspectos relacionados con la enseñanza del CAD en la Facultad.

El procedimiento seguido fue:

1. Formación de equipos de trabajo entre los integrantes del proyecto.

2. Determinación de las variables a estudiar.

3. Recolección de información.

4. Análisis de la tecnología CAD.

5. Diagnóstico de la enseñanza CAD en la Facultad.

6. Presentación de aplicaciones CAD en las asignaturas.

7. Propuesta de software, hardware, base de datos CAD y humanware.

Las variables utilizadas fueron:

1. Información disponible sobre la tecnología CAD.

2. Software $C A D$ al alcance de los recursos de la universidad.

3. Hardware necesario para trabajar en los laboratorios con software CAD.

4. Humanware o recursos humanos con que se cuenta en la facultad en lo relacionado al conocimiento y manejo de la tecnología CAD.

5. Enseñanza de temas relacionados al CAD en las asignaturas de la Facultad, en el semestre 2005-I.
6. Aplicaciones CAD en las asignaturas del plan de estudios.

\section{DIAGNÓSTICO DEL CAD EN LA EAPII}

1. Información encontrada sobre la tecnología CAD:

a. Librerías: Ios libros más actualizados se encuentran en inglés y se compran mediante pedido; en castellano sólo se pudo encontrar libros con 2 años de antigüedad de edición.

b. Revistas: en el mercado nacional no se elabora ninguna revista relacionada con el tema.

c. En la biblioteca de la Facultad no existen libros, ni revistas actualizadas.

d. Internet: usando los buscadores se puede encontrar mucha información especialmente de tipo comercial, existiendo poca información académica.

e. En las bibliotecas de la universidad no existen libros actualizados.

2. La tecnología CAD en nuestro medio, se encuentra orientada a las siguientes áreas:

a. Modelado del objeto y su representación ortogonal.

b. Representación foto realista, animación y simulación.

c. Cálculo mediante elementos finitos.

d. Cálculos aplicando algoritmos particulares.

3. Los software CAD más usados por las empresas industriales son:

a. Software para el dibujo técnico: Autocad, Mechanical Desktop, Inventor, Solid Edge y Solid Work.

b. Software para las áreas de mecánica y resistencia de materiales: Mechanical Desktop Pack, Solid Work cosmos, Inventor y SAP.

c. Software para el área de procesos de manufactura: Surfcam y Edge Cam

d. Software para el manejo de sistemas de información georefenciadas: Map Info, Arc View, Autocad Map y Microstacion.

Los nuevas versiones de los software CAD trabajan bajo la plataforma Windows XP o similar.

4. Los softwares CAD que cuenta la Facultad:

a. En el año 1999, RECOMAC instaló en un computador del Laboratorio de Máquinas y Herramientas el software Surfcam.

b. En el año 2001 se compraron 12 licencias del software Mechanical Desktop V6, para Autocad 2002.

c. En el año 2001, se recibió en donación el software Nastram, una licencia.

Los softwares Nastram y Surfcam no fueron 
Cuadro 1. Cursos de la EAPII con temas relacionados al CAD

\begin{tabular}{|c|c|c|c|c|c|c|c|}
\hline \multirow{2}{*}{$\begin{array}{l}\text { Cursos que se pueden } \\
\text { desarrollar temas CAD }\end{array}$} & \multirow{2}{*}{ Ciclo } & \multirow{2}{*}{$\begin{array}{l}\text { Crédi- } \\
\text { tos }\end{array}$} & \multicolumn{3}{|c|}{ HORAS } & \multicolumn{2}{|c|}{ Nivel aplicación } \\
\hline & & & Teoría & Práct. & Laborat. & Teórico & Softw. \\
\hline Dibujo Técnico & 1 & 4.0 & 2 & 3 & 1 & --- & $\mathrm{Si}$ \\
\hline Física I & 2 & 5.0 & 3 & 2 & 2 & --- & --- \\
\hline Geometría Descriptiva & 2 & 4.0 & 2 & 4 & 0 & --- & --- \\
\hline Mecánica Aplicada & 4 & 5.0 & 4 & 2 & 0 & --- & --- \\
\hline Métodos Computacionales & 4 & 5.0 & 3 & 2 & 2 & --- & --- \\
\hline Resistencia de Materiales & 5 & 4.0 & 3 & 2 & 0 & ---- & --- \\
\hline Dibujo Industrial & 5 & 3.5 & 1 & 3 & 2 & $\mathrm{Si}$ & $\mathrm{Si}$ \\
\hline Ingeniería de Materiales & 5 & 4.0 & 3 & 2 & 0 & --- & --- \\
\hline Ergonomía & 6 & 4.0 & 3 & 2 & 0 & --- & --- \\
\hline $\begin{array}{l}\text { Procesos de Manufactura } \\
\text { I }\end{array}$ & 6 & 4.0 & 3 & 2 & 0 & $\mathrm{Si}$ & --- \\
\hline Distribución de Planta & 7 & 3.0 & 2 & 2 & 0 & --- & --- \\
\hline Logística & 7 & 3.0 & 2 & 2 & 0 & $\mathrm{Si}$ & --- \\
\hline Tecnología Industrial & 9 & 3.5 & 2 & 2 & 1 & --- & $\begin{array}{ll}-- \\
\end{array}$ \\
\hline $\begin{array}{l}\text { Modelación y Simulación } \\
\text { Sistemas }\end{array}$ & 9 & 3.5 & 2 & 0 & 2 & $\mathrm{Si}$ & --- \\
\hline
\end{tabular}

usados, ningún profesor le dedicó tiempo a aprenderlo, analizarlos y aplicarlos.

Después de esto no se ha actualizado y/o comprado software alguno.

Los software con que se trabaja en los laboratorios son con permisos temporales por lo que son incompletas y sin manuales; esto dificulta mucho el trabajo académico.

6. Hardware en la Facultad:

a. El el año 2001 el Laboratorio CAD de la FII, contaba con 15 computadoras Pentium 3 RAM 128 y un plotter Hewlett Packard D500 A0. A la fecha sólo 4 computadoras están operativas y no han sido repotenciadas.

b. Los laboratorios de los cursos de dibujo se vienen dictando en el laboratorio de informática, se cuenta con 24 computadores P4, con 256MB. (normalmente un promedio de 20 se encuentran operativas).

7. Humanware:

a. Con respecto al manejo de software: dibujo asistido por computador, de 10 profesores que dictan las asignaturas relacionadas con el di- bujo, 3 tienen conocimientos intermedios y 6 de ellos sólo tienen conocimientos básicos.

b. Con respecto al manejo de software: diseño asistido por computador sólo hay 2 profesores con conocimientos básicos.

c. El círculo de estudios CAD (CIDECAD) formado con el objetivo de desarrollar aplicaciones $C A D$, en la fecha se encuentra reducido a un pequeño grupo de alumnos, no cuenta con los recursos necesarios, y no tienen apoyo de las autoridades de la Facultad.

8. Base de datos: con el grupo CIDECAD en el año 2001 se comenzó a implementar una hemeroteca con 5 libros/manuales relacionados al CAD, se publicaron 5 separatas sobre Autocad, Mechanical Desktop y 3D Studio y mensualmente se emitieron boletines; en los últimos 4 años se ha realizado pequeños aportes aislados. Hasta la fecha sólo un profesor realizó 3 publicaciones de separatas para las clases de laboratorios y una presentación en Power Point de ejercicios desarrollados.

9. En el cuadro 1 se presenta las asignaturas del plan de estudios de la EAPII en los que se pue-

Cuadro 2. Software CAD/CAE usados en los cursos de la EAPII

\begin{tabular}{|l|l|l|l}
\hline Curso & Software Usado & Tema tratado & Comentario \\
\hline $\begin{array}{l}\text { Dibujo } \\
\text { Técnico }\end{array}$ & AutoCAD & Dibujo 2 dimensiones & Desarrollo básico \\
\hline $\begin{array}{l}\text { Dibujo } \\
\text { Industrial }\end{array}$ & Mechanical Desktop & $\begin{array}{l}\text { Dibujo 3 dimensiones } \\
\text { Cálculo con elementos finitos. }\end{array}$ & $\begin{array}{l}\text { Desarrollo intermedio } \\
\text { Desarrollo básico }\end{array}$ \\
\hline
\end{tabular}


Cuadro 3. Software CAD/CAE presentados en conferencias

\begin{tabular}{|l|l|l|c|}
\hline Curso & Software Usado & Tema Tratado & Nro. Sesiones \\
\hline Dibujo & Inventor & Dibujo 3 dimensiones & 2 \\
Industrial & Solid Work & Dibujo 3 dimensiones & 1 \\
& Solid Edge & Dibujo 3 dimensiones & 1 \\
& Solid Work Cosmos & Dibujo 3 dimensiones & 1 \\
& 3D Studio & Animación & 2 \\
& SAP & Cálculo estructuras & 1 \\
& Map Info & Planos georeferenciados & 1 \\
\hline
\end{tabular}

den tratar temas relacionados a la tecnología CAD, así como el ciclo correspondiente en que se dictan, créditos del curso, horas de clase y su nivel de aplicación (teórico o con aplicación de algún software).

Como se puede apreciar las horas de laboratorio dedicadas al uso efectivo de software CAD en todo el plan de estudios es de 03 horas semanales.

10. Software CAD usados en la EAPII semestre 2005-I (ver cuadro 2).

11. Software CAD usados a nivel de conferencias (ver cuadro 3$)$.
12. El software AutoCAD es usado para el trabajo en gráficos de dos dimensiones, como planos, esquemas, etc. En la figura 1 se presenta un gráfico desarrollado en ese software.

13. El software Mechanical Desktop es usado en el modelamiento (ver figura 2) y en la representación de vistas múltiples (ver figura 3). A pesar de ser reemplazado por el Inventor es muy usado en el medio industrial.

14. Software CAD propuestos para ser adquiridos y aplicados en las asignaturas de la EAPII para el año 2006.

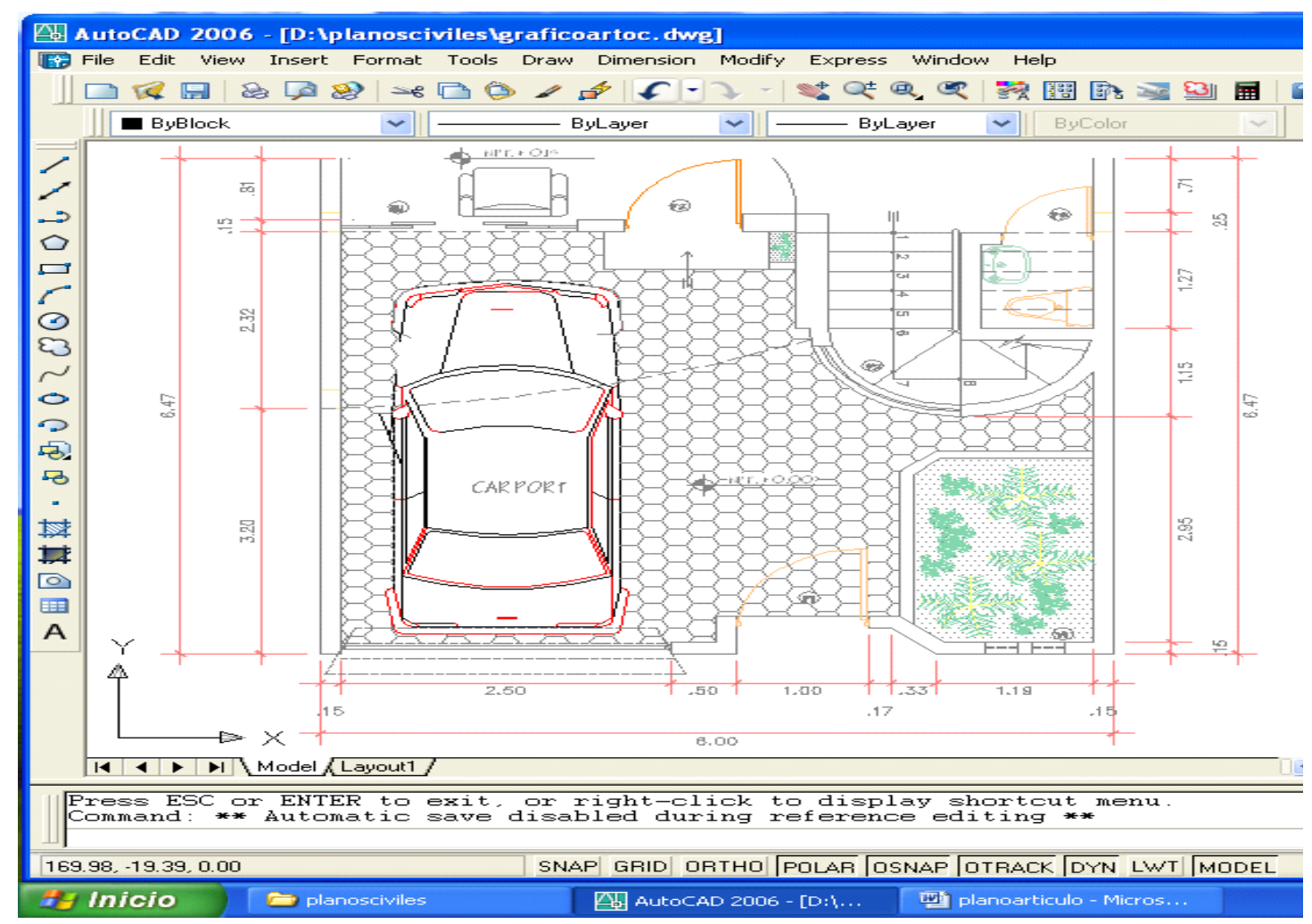

Figura 1. Aplicación del software Autocad (trabajo en dos dimensiones) Fuente: Elaboración propia, 2006. 


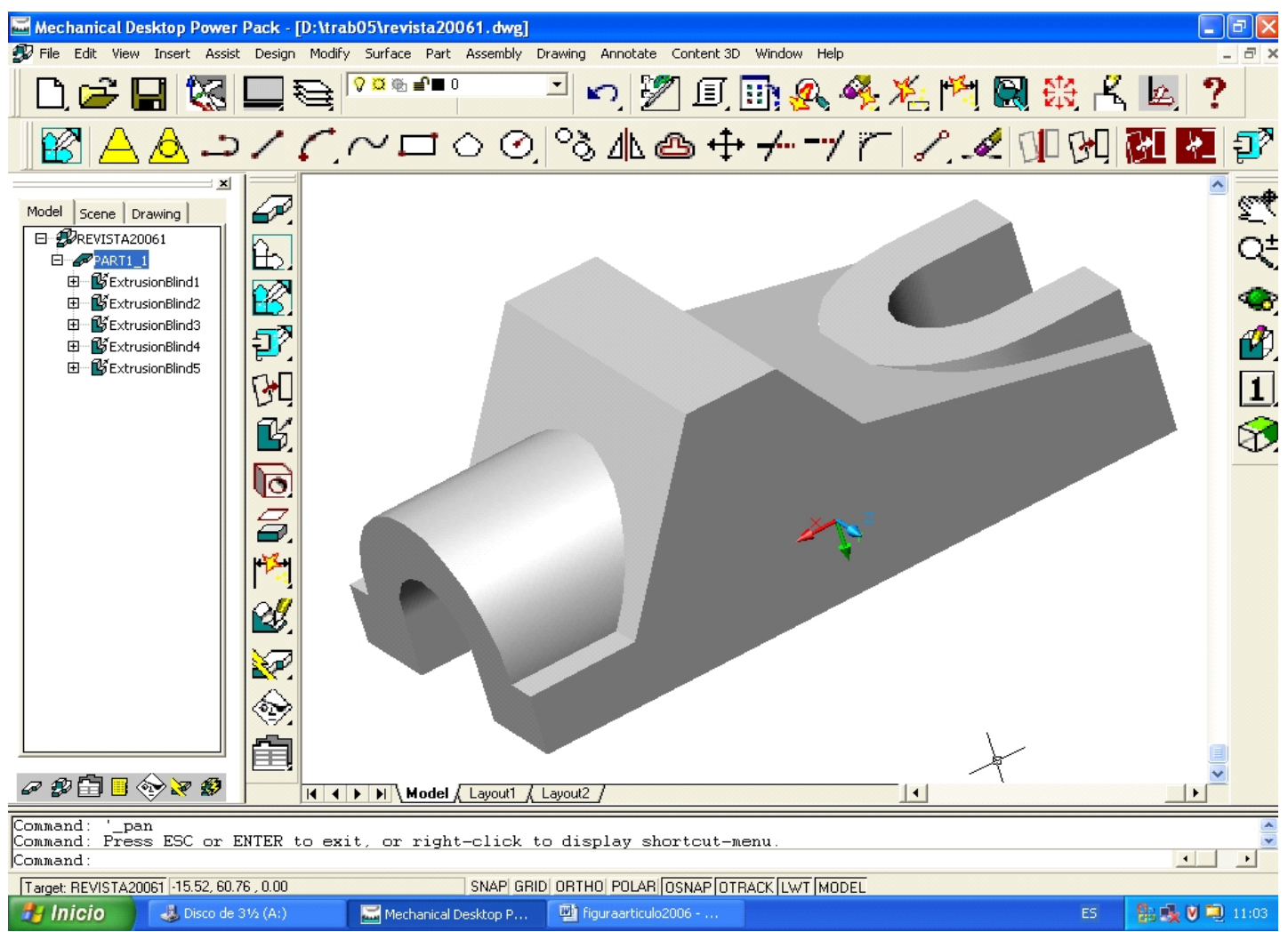

Figura 2 . Aplicación del software Mechanical Desktop (modelado sólido 3D) Fuente: Elaboración propia, 2006.

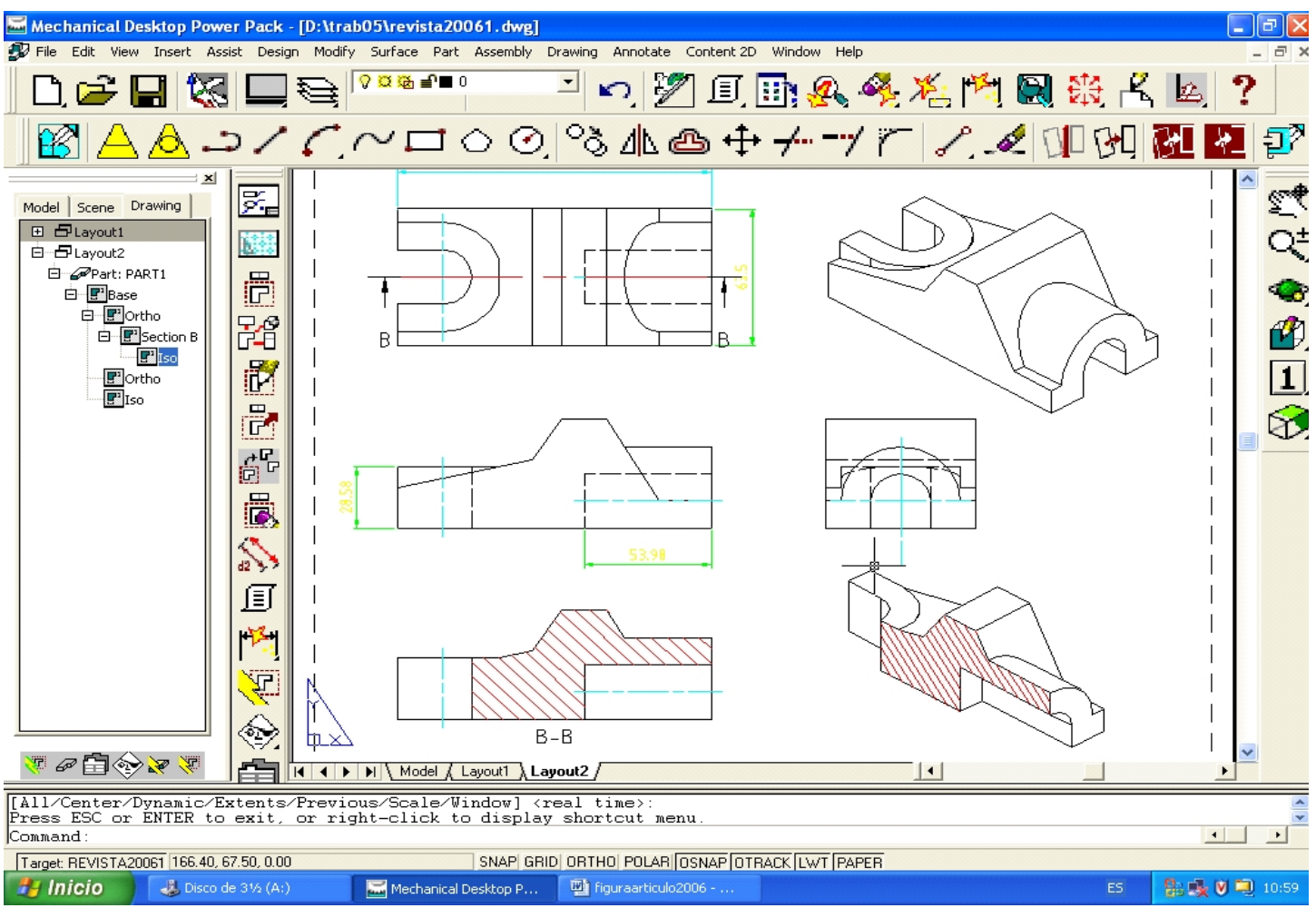

Figura 3. Aplicación del software Mechanical Desktop (vistas múltiples-layout) Fuente: Elaboración propia, 2006. 
Cuadro 4. Softwares CAD propuestos

\begin{tabular}{|c|c|c|}
\hline Curso & Tema & Software \\
\hline Dibujo Técnico & $\begin{array}{l}\text { Dibujo } 2 \text { dimensiones } \\
\text { Modelamiento de sólidos } \\
\text { Secciones básicas }\end{array}$ & $\begin{array}{l}\text { Autocad V2006 } \\
\text { Inventor } 2006\end{array}$ \\
\hline Geometría Descriptiva & $\begin{array}{c}\text { Vistas auxiliares } \\
\text { Intersección plano/sólido } \\
\text { Intersección de sólidos } \\
\text { Desarrollos }\end{array}$ & $\begin{array}{l}\text { Inventor } 2006 \\
\text { SPI-Sheetmetal }\end{array}$ \\
\hline Mecánica Aplicada & Cálculo de estructuras & SAP 2004 \\
\hline Resistencia de Materiales & $\begin{array}{c}\text { Cálculo de cargas } \\
\text { Cálculo con elementos finitos }\end{array}$ & $\begin{array}{c}\text { Solid Work } 2005 \\
\text { Nastran for Windows }\end{array}$ \\
\hline Dibujo Industrial & $\begin{array}{c}\text { Ensamble y Despiece } \\
\text { Planos de edificación } \\
\text { Animación } \\
\end{array}$ & $\begin{array}{l}\text { Inventor } 2006 \\
\text { Architectural desktop } 2006 \\
\text { 3D studio Viz R2 } \\
\end{array}$ \\
\hline $\begin{array}{l}\text { Procesos de Manufactura } \\
\text { I }\end{array}$ & Procesos de arranque de viruta & $\begin{array}{c}\text { Surfcam } 2005 \\
\text { Edge Cam } 2006\end{array}$ \\
\hline Distribución de Planta & Visualización de espacios & Autodesk VIZ 2006 \\
\hline Logística & Sistemas georeferenciados & Map info V8 \\
\hline
\end{tabular}

\section{APORTE E IMPORTANCIA CIENTÍIICA TECNOLÓGICA}

El aporte de este estudio es presentar las técnicas del desarrollo CAD y conocer como se viene dictando estas tecnologías en el plan de estudios de la EAPII así como presentar una propuesta de temas a ser incluidos.

\section{CONCLUSIONES Y RECOMENDACIONES}

El sistema CAD se viene desarrollando en forma acelerada y debido al desarrollo del software y hardware su aplicación es de uso extensivo. Las universidades de los países en desarrollo deben adoptar estas tecnologías, por lo que la inclusión de estos temas en la currícula permitirá mejores la formación del futuro ingeniero industrial.

La carencia de software, hardware, base de datos y humanware no permite la aplicación de las tecnologías CAD en las asignaturas de la EAPII.

El hardware básico para un laboratorio donde se desarrollan las prácticas CAD debería tener:

a. Intel Pentium 4, 4GHz o equivalente.

b. Microsott Windows XP professional - Windows 2000 -Windows NT 4.0

c. $512 \mathrm{MB}$ de RAM

d. $650 \mathrm{MB}$ de espacio libre en disco y $75 \mathrm{MB}$ de espacio de intercambio

e. Monitor VGA de $1024 \times 768 \times 32 \mathrm{~K}$

f. Tarjeta de video 3D AGP-256MB

g. Unidad DVD, CD-RW 52X32X52

h. Mouse con wheel.
Se debe buscar el aprovechamiento de recursos de la universidad y formar un laboratorio CAD/CAM para todas las ingenierías, de manera que se esté actualizado en el desarrollo de software y hardware.

Orientar los destinos de la universidad como un ente educativo vinculado con el sector empresarial y la sociedad a fin de intercambiar tecnologías, soluciones y conocimientos; esto se puede lograr desarrollando trabajos aplicativos de campo.

Relacionar estas tecnologías con los cursos impartidos en la Facultad mediante el uso de los laboratorios y centros de producción, buscando aplicaciones directas e innovaciones en cada uno de ellos.

En el diseño y análisis de componentes mecánicos la práctica nos dice que sí es viable una solución analítica y/o experimental a un bajo costo y con alto nivel de confiabilidad.

La edad promedio de los profesores del área de dibujo pasa los 50 años, por lo que es necesario contratar docentes jóvenes (con amplio dominio de las técnicas CAD/CAE) para el dictado de los laboratorios de los cursos relacionados con el diseño.

Reorientar el trabajo desarrollado por el CIDECAD, dotándole del equipamiento necesario, de manera que sea el ente que elabore manuales, guías de práctica, boletines, servicios a terceros, etc.

Encargar a los docentes la elaboración de manuales de laboratorio de las asignaturas que trabajan con técnicas CAD. 
En el desarrollo de los trabajos CAD se debe usar las técnicas de la ingeniería concurrente vía red con procesamiento colaborativo.

\section{REFERENCIAS BIBLIOGRÁFICAS}

1. Bertoline G., Millar C., y Mohler J., Wiebe E. (1999) Dibujo en Ingeniería y Comunicación Gráfica. $2^{\mathrm{a}}$. Ed., McGraw-Hill, México.

2. Cincunegui D. (1999) CADXPress. Ingeniería asistida. 3(15): 20-22, México.
3. Computeraided engineering, USA, www.caenet.com Julio 2005.

4. Cuellar J.(2000) CADXPress. Ingenieros digitales. 4(25): 36-39, México.

5. Dixon John B. (1979) Diseño en Ingeniería. 1ª Ed. Limusa, México.

6. Gould L. (1998) Metalmecánica Internacional. 1(3): 22-25, Colombia.

7. Trotz L, Poletillo J. (1999) CADXPress. Ingeniería asistida. 3(15): 24-31, México. 